\title{
Shades of grey: the need for a multi-disciplinary approach to research investigating alcohol and ageing
}

\author{
Celia Wilkinson, ${ }^{1,2}$ Julie Dare ${ }^{1}$ \\ ${ }^{1}$ Edith Cowan University, Joondalup; ${ }^{2}$ National Drug Research Institute, Curtin University, Perth, \\ Australia
}

Significance for public health

With the rapid ageing of the global population and concerns about recent increases in the consumption of alcohol amongst older people, the issue of alcohol and ageing is becoming an important public health issue. However, there remains little research that adopts a holistic multi-disciplinary perspective. Such research is important and may offer the best way forward in terms of unravelling the complexity of competing risks and benefits associated with low to moderate drinking amongst older people.

\begin{abstract}
This paper calls for an increase in multi-disciplinary research on the issue of alcohol and ageing, to ensure public health interventions reflect the complex and diverse needs of older drinkers. Older people (65+ years) represent a unique segment of the population; compared to adolescents and younger people, they are more likely to have a range of co-morbid conditions and be taking prescribed medication, and are more physiologically vulnerable to the effects of alcohol. This suggests that from a public health perspective, alcohol use by older people is problematic. However, as with younger people, alcohol use is closely associated with socialisation and social engagement. While social engagement is important at all stages of life, it is particularly critical as people age, when many of the formal social roles which provide a catalyst for social integration shift or are lost. Currently, however, there is little evidence of an integrated public health response to the complex issue of alcohol and ageing. That is, what is needed is a concurrent acknowledgement of the health problems that may be associated with contraindicated alcohol use, versus the potential health benefits that can accrue from social drinking. This will require a holistic rather than reductionist approach that integrates biomedical and social science insights to develop a more comprehensive and nuanced understanding of the implications of alcohol use amongst diverse populations of older people.
\end{abstract}

\section{Introduction}

The ageing of the global population presents significant public health challenges, and has prompted research from a range of disciplines with the aim of developing an evidence base on how best to facilitate healthy ageing. However, much research has operated from a single discipline or single outcome perspective, and as such, opportunities to view the process of healthy ageing from a more holistic position have been lost.

One area where this singular focus is often evident is research investigating alcohol use amongst older people (65+ years). Research in this area has for the most part adopted a biomedical focus, and been characterised by a concern with the negative consequences of higher levels of alcohol consumption amongst older people. This concern is compounded by evidence of higher levels of alcohol consumption amongst later generations of older people. ${ }^{1}$ While research reflecting a biomedical focus has been vital in understanding the risks associated with hazardous levels of alcohol use, it has rarely examined the role that low to moderate (defined as 10 to 20 grams of ethyl alcohol per day) levels of drinking may play in social engagement, and by extension positive outcomes in health and wellbeing. ${ }^{2}$

Similarly, while social science research has focused on links between social engagement and health and wellbeing, it has failed to disaggregate the possible role of low to moderate levels of consumption as a social lubricant. In this context, alcohol may be instrumental in facilitating social engagement for some older people. ${ }^{3}$ Conversely, little research has considered whether engagement in social activities may also drive or motivate alcohol use amongst older people.

These contrasting research activities suggest a disconnect between the social science and biomedical disciplines publishing in the area of alcohol and ageing. The research strands seem to work most often in parallel, with only rare reference to other disciplinary bodies of knowledge, or the insights they may offer. And yet, as Hennessy and Walker noted, ageing is a complex phenomenon that can best be understood by adopting a multi-disciplinary perspective that draws together research and literature across the social, medical and biological sciences. ${ }^{4}$

The aim of this paper is to draw attention to the complex role alcohol plays in many older people's lives, and to advocate for the adoption of a comprehensive multi-disciplinary approach to public health research, particularly in relation to examining potential associations between moderate alcohol use, social engagement, subjective wellbeing and the process of healthy ageing amongst diverse populations of older people.

The paper begins by considering the issue of drinking in moderation, and then provides a brief overview of some of the literature on biomedical harms and benefits associated with alcohol use amongst older people. This is followed by an analysis of potential psychosocial benefits associated with moderate alcohol use amongst older people, and a discussion on the degree to which moderate alcohol use may facilitate social engagement. The paper concludes by examining the implications for public health research investigating alcohol use amongst older people.

\section{Moderate drinking}

One of the difficulties in the area of alcohol and ageing is determining what levels of consumption represent moderate drinking and what levels represent hazardous or harmful levels. Achieving consensus on a universal definition of moderate drinking has proven elusive. ${ }^{5}$ One of 
the difficulties with developing a definition of what constitutes moderate drinking for older people is that they are more likely than young people to have a range of co-occurring medical conditions, be on medication, and because of the physiological changes that occur with ageing, have increased sensitivity to the effect of alcohol. ${ }^{6}$ While some countries have now developed drinking guidelines specifically for older people, there is still the need for further work and universal consensus on what might represent moderate drinking for older people, as this has a plethora of public health implications. In the context of this paper, and while acknowledging that much of the following research often does not quantify low, moderate or high levels of consumption, moderate drinking has been used by the authors to describe up to 20 grams of ethyl alcohol per day (equivalent to 2 standard drinks in Australia). ${ }^{2}$

\section{Biomedical consequences associated with alcohol use amongst older people}

As people age they become increasingly vulnerable to a range of medical conditions. However, alcohol consumption adds a further compounding factor, ${ }^{2}$ due to age related physiological changes including a decrease in total body water, and lean body mass. ${ }^{6}$ Research has identified a myriad of complex and often competing risks and benefits associated with both the short and long term use of alcohol amongst older people. For example, some of the short term harms associated with alcohol use amongst older people include falls, motor vehicle crashes, pedestrian fatalities and adverse drug reactions. ${ }^{7}$ In addition, given that alcohol has now been listed as a Group 1 carcinogen, ${ }^{8}$ it is likely that older people who have been drinking for decades may have an elevated risk of developing alcohol-related cancers. This evidence suggests that older people, and in particular older women because of greater physiological sensitivity, are more likely to be at risk of alcohol related trauma at even relatively low levels of consumption. ${ }^{2}$

Conversely, there is also research supporting a raft of important potential benefits for older people from moderate levels of alcohol use. For example, there is evidence that 10 to 30 grams of ethyl alcohol may have a protective effect against heart disease. ${ }^{9}$ In addition, an Australian study indicated that men who consumed up to 40 grams of ethyl alcohol per day and women who consumed up to 20 grams per day and had up to two alcohol free days per week, had a reduced risk of mortality over the next five years. ${ }^{10}$ In a similar vein, research from the United States indicated that women aged 65 years and older who drank up to 24 grams of ethyl alcohol per day had better self reported health. ${ }^{11}$

The complexity of alcohol-related net benefits and harms is well illustrated in the research literature examining alcohol and cognitive function. While lower cognitive function has been reported amongst non drinkers compared to drinkers, ${ }^{12}$ and a $j$ or $u$ shaped relationship has been found between levels of consumption and cognitive function, ${ }^{13}$ there is also a body of literature indicating no significant relationship between alcohol and cognitive function, ${ }^{14}$ as well as research which suggests that alcohol may delay age associated cognitive decline in women but not in men. ${ }^{15}$

The influence of alcohol on a person's health will also vary depending on a range of factors, including environmental and genetic factors, and the frequency, duration and amount of alcohol consumed. According to Gross and colleagues, ${ }^{14}$ some of the inconsistencies apparent in the associations between alcohol and cognition may reflect methodological differences in how cognition is measured and how alcohol use is quantified. The suggested association between moderate alcohol use and improved cognition may also be an artefact due to confounding of pre-morbid intellect and physical health, demographic fac- tors, or factors related to the social context in which alcohol is consumed. ${ }^{16}$

The links between social context and alcohol use amongst older people are particularly relevant to investigations around alcohol and cognition, given evidence that social engagement may have a protective effect on cognitive function and dementia. ${ }^{17}$ For example, research indicates that a more active lifestyle and greater involvement in leisure activities are protective of late-life cognitive decline and dementia risk. ${ }^{18}$ The extent of this protection was highlighted in a longitudinal study conducted with a cross-cultural sample in the United States, which found that a larger personal social network reduced the rate of cognitive decline by $39 \%$ when compared to smaller social networks, and high social involvement reduced this decline by over $90 \%{ }^{19}$

While researchers investigating alcohol use amongst older people have hinted at the possible confounding nature of psychosocial factors such as social engagement, rarely have such variables been controlled for or considered in any detail when analysing the sometimes conflicting outcomes often seen in the alcohol literature. Moreover, while variables such as quantity and frequency of alcohol consumption, socioeconomic status, gender, ethnicity, marital or partner status, and education are often controlled for, the social context in which drinking takes place is typically overlooked as a potential confounder. Indeed, in contrast to the relatively large body of research on the biomedical consequences of alcohol use, the psychosocial dimensions of drinking have received much less attention. ${ }^{20}$

What research has been conducted in this area has for the most part focused on problem or at risk drinking amongst older people, such as the impact of problem drinking on older people's psychological health, ${ }^{21}$ or links between stressful life events and excessive or problem drinking. ${ }^{22}$ Such research has highlighted older people's use of alcohol as a social prop to help them cope with role changes, loneliness, isolation or boredom. ${ }^{1}$

In addition, research examining the links between alcohol use and psychosocial wellbeing has generally relied upon quantitative research instruments. As a result, they often provide limited insights into the social dimensions of older people's alcohol use, and have not explored in any detail the potential links between alcohol use, social engagement and older people's health and subjective wellbeing. Significantly, the few studies which have used qualitative methodologies such as ethnography ${ }^{23}$ and in-depth interviews ${ }^{3,24}$ to explore this area provide rich insights into links between alcohol use and culture and social meaning, and cohort and life-course. Qualitative research with different cultural groups has highlighted implicit conventions around the acceptable use of alcohol by older people, which are in turn shaped by not only the individual's previous drinking career, ${ }^{24}$ but also their social circle and cultural norms. ${ }^{3,23,24}$ While these norms may differ across cultures, what appears common is the often hedonistic use of alcohol by older people in cultures and countries where alcohol use is permitted and acceptable, $, 23,24$ as demonstrated through comments such as: it's fun when you are out in a nice place in good company, then it's fun to have. ${ }^{24}$ Perspectives such as these, which highlight the conviviality and pleasure associated with social drinking, are difficult to capture in purely quantitative research, and yet are critical to understanding the benefits older people gain through alcohol use, and hence help to explain why they drink.

\section{Older people, social engagement and alcohol}

Perhaps not surprisingly, the links between psychosocial factors such as social engagement and social connectedness, and health and wellbeing outcomes have received considerable attention in the social 
sciences. For example, a compelling body of evidence has documented how psychosocial factors influence physiological health, ${ }^{25}$ cognitive function, ${ }^{17}$ self esteem, ${ }^{26}$ and subjective wellbeing and quality of life. ${ }^{26}$

Social engagement appears to be particularly important for older people, as many of the formal social roles which provide a catalyst for social integration, shift or are lost as people age. Factors commonly associated with ageing, including retirement, bereavement, living alone, care-giving responsibilities, restricted mobility and declining health, ${ }^{27}$ can impact on older people's self-identity and potentially increase their risk of social isolation. ${ }^{28}$ Consequently, participation in activities fostering social engagement and integration are key determinants of healthy ageing.

As with other age groups, higher levels of social engagement amongst older people are often associated with alcohol use. ${ }^{3}$ Indeed, sociability is one of the main reasons offered by older people to explain their use of alcohol..$^{29}$ Arguably, settings which promote regular social activities, such as purpose built retirement communities, may facilitate increases in the frequency and/or consumption of alcohol consumption amongst residents.

There is some evidence to support this suggestion, with research conducted in retirement communities in the United States and Australia identifying high levels of social engagement and regular alcohol use amongst residents. ${ }^{3,30}$ For example, a recent Australian study found that during village-based social activities it was most common for almost everybody attending to consume alcohol. ${ }^{3}$ As a 67 year old female participant explained: drinking is a social thing and a lot of people don't have a drink by themselves, and here there are a lot of social activities. ${ }^{3}$ Increases in alcohol consumption were explained by participants as being related to their extra social involvement since moving to the retirement village, and the freedom from having to drive home after village-based social activities. ${ }^{3}$

This evidence offers important insights into the social dimensions of alcohol use amongst older people, and highlights the degree to which older people's alcohol use can be closely connected with high levels of social engagement and interaction. It also draws attention to the possibility that older people's social alcohol use may confer benefits such as psychological and social well-being, which are important health outcomes in their own right. ${ }^{31}$

\section{Public health implications}

As this paper indicates, different research streams have identified risks and benefits associated with differing levels of alcohol consumption amongst older people. However, despite the need for research investigating the health outcomes associated with alcohol use to take account of psychosocial factors such as social engagement, ${ }^{31}$ little remains published in this area. This suggests a continuing disconnect between biomedical and social science perspectives, compounded by different disciplines working in relative isolation. Research in the alcohol field has been largely concerned with the effect of varying levels of ethyl alcohol on biomedical health, without often considering how social factors associated with alcohol may confound or explain results. Similarly, much social science research has focused on the social processes supporting health, without necessarily examining the role alcohol may play in facilitating or constraining social engagement, and the associated influences on health. This is not a criticism of any discipline or research field; as such research is important in developing an evidence base about how best to support healthy ageing. It does, however, suggest that integration of different research perspectives will offer the best opportunity for developing deeper insights into issues related to alcohol and older people.

The ageing of the global population, along with evidence that younger cohorts of older people drink alcohol at higher levels than their predecessors, ${ }^{1}$ has important implications for public health. For example, high levels of polypharmacy are common amongst older people, ${ }^{32}$ as are alcohol-related adverse drug reactions. ${ }^{33}$ In addition, older heavier drinkers are less likely to access recommended preventive health services, ${ }^{34}$ and yet are frequently admitted to emergency departments, ${ }^{35}$ placing greater demand on already over-stretched tertiary health services. In responding to these issues, a number of authors have recommended that, for example, screening of older people's alcohol use needs to occur more frequently and systematically. ${ }^{34}$ While concurring with this, it is also important that public health responses to reduce alcoholrelated harms in older populations do not overlook the significant biopsycho-social benefits which may accrue from moderate levels of drinking. As such, an important caveat is that older people's alcohol use needs to be viewed not solely through a biomedical lens, but rather from a holistic perspective which acknowledges the complex role alcohol plays in many older people's lives.

Research conducted with older people serves as a reminder that individuals are social beings who influence and are influenced by their environment, culture and their traditions. This highlights that public health strategies need to acknowledge that older people are not homogenous and nor is their alcohol use. Public health research which brings together multi-disciplinary perspectives has the potential to add another layer to our understanding of the consequences of low to moderate alcohol use amongst older people.

\section{Conclusions}

Investigation and management of alcohol use amongst older people is becoming increasingly important as a public health issue. However, while more biomedical research is needed, so too is research that contextualises alcohol use and examines a range of psychosocial measures as both outcomes and confounders. Echoing recent calls for a greater multi-disciplinary approach to research on ageing, ${ }^{4}$ we contend that a richer understanding of the harms and benefits of alcohol use amongst older people can be achieved by drawing together a range of research perspectives. While alcohol use amongst older people raises a number of challenging public health issues, the adoption of a multi and interdisciplinary approach offers significant opportunities for better understanding the complex role alcohol plays in an active and sociallyengaged ageing population.

Correspondence: Celia Wilkinson, Edith Cowan University, 270 Joondalup Drive, Joondalup, WA, 6027 Australia. Tel. +61.863.042597.

E-mail: c.wilkinson@ecu.edu.au

Key words: aged, alcohol, elderly, social engagement, multi-disciplinary. Contributions: the authors contributed equally.

Conflict of interests: the authors declare no potential conflict of interests.

Received for publication: 8 July 2013.

Revision received: 10 October 2013.

Accepted for publication: 16 October 2013.

CCopyright C. Wilkinson and J. Dare, 2014

Licensee PAGEPress, Italy

Journal of Public Health Research 2014; 3:180

doi:10.4081/jphr.2014.180

This work is licensed under a Creative Commons Attribution NonCommercial 3.0 License (CC BY-NC 3.0). 


\section{References}

1. Smith L, Foxcroft D. Drinking in the UK: an exploration of trends. London: Joseph Rowntree Foundation; 2009.

2. Wilkinson C, Lintzeris N, Haber P. Special populations: older people. Canberra: Australian Government Department of Health and Ageing; 2009.

3. Wilkinson C, Dare J, Waters S, et al. An exploration of how social context and type of living arrangement are linked to alcohol consumption amongst older Australians. Joondalup: Edith Cowan University, School of Exercise and Health Sciences; 2012.

4. Hennessy $\mathrm{CH}$, Walker A. Promoting multi-disciplinary and interdisciplinary ageing research in the United Kingdom. Ageing Soc 2011;31:52-69.

5. Furtwaengler NA, De Visser R0. Lack of international consensus in low-risk drinking guidelines. Drug Alcohol Rev 2013;32:11-8.

6. Dufour MC, Fuller RK. Alcohol in the elderly. Ann Rev Med 1995;46:123-32.

7. Chikritzhs T, Pascal R. Trends in alcohol consumption and related harms for Australians aged 65 to 74 years (the young-old), 19902003, National Alcohol Indicators. Perth, Australia: National Drug Research Institute, Curtin University of Technology; 2005.

8. International Agency for Research on Cancer World Health Organization. Breast and colorectal cancers are associated with cancer. Press release No. 175. Geneva, Switzerland: World Health Organization 2007. 2 November 2010. Available from: http://www.iarc.fr/en/media-centre/pr/2007/pr175.html.

9. Snow WM, Murray R, Ekuma 0, et al. Alcohol use and cardiovascular health outcomes: a comparison across age and gender in the Winnipeg Health and Drinking Survey Cohort. Age Ageing 2009;38:206-12.

10. McCaul KA, Almeida OP, Hankey GJ, et al. Alcohol use and mortality in older men and women. Addiction 2010;105:1391-400.

11. Balsa AI, Homer JF, Fleming MF, French MT. Alcohol consumption and health among elders. Gerontologist 2008;48:622-36.

12. Lindeman RD, Wayne S, Baumgartner RN, Garry PJ. Cognitive function in drinkers compared to abstainers in the New Mexico Elder Health Survey. J Gerontol A Biol Sci Med Sci 2005;60A:1065-70.

13. Weyerer S, Schäufele M, Wiese B, et al. Current alcohol consumption and its relationship to incident dementia: results from a 3-year follow-up study among primary care attenders aged 75 years and older. Age Ageing 2011;40:456-63.

14. Gross AL, Rebok GW, Ford DE, et al. Alcohol consumption and domain-specific cognitive function in older adults: longitudinal data from the Johns Hopkins Precursors Study. J Gerontol B Psychol Sci Soc Sci 2011;66B:39-47.

15. Stott DJ, Falconer A, Kerr GD, et al. Does low to moderate alcohol intake protect against cognitive decline in older people? J Am Geriatr Soc 2008;56:2217-24.

16. Anstey KJ, Windsor TD, Rodgers B, et al. Lower cognitive test scores observed in alcohol abstainers are associated with demographic, personality, and biological factors: the PATH Through Life Project. Addiction 2005;100:1291-301.

17. Glei DA, Landau DA, Goldman N, et al. Participating in social activ- ities helps preserve cognitive function: An analysis of a longitudinal, population-based study of the elderly. Int $\mathrm{J}$ Epidemiol 2005;34:864-71.

18. Engelhardt H, Buber I, Skirbekk V, Prskawetz A. Social involvement, behavioural risks and cognitive functioning among older people. Ageing Soc 2010;30:779-809.

19. Barnes LL, Mendes de leon CF, Wilson RF, Bienias JL, Evans DA. Social resources and cognitive decline in a population of older African Americans and whites. Neurology 2004;63:2322-6.

20 . Heath $D$. Why we don't know more about the social benefits of moderate drinking. Ann Epidemiol 2007;17:71-4.

21. St. John PD, Montgomery PR, Tyas SL. Alcohol misuse, gender and depressive symptoms in community-dwelling seniors. Int J Geriatr Psychiatry 2009;24:369-75.

22. Brennan PL, Moos BS. Late-life drinking behavior. Alcohol Health Res W 1996;20:197-204.

23. Moolasart J, Chirawatkul S. Drinking culture in the Thai-Isaan context of north-east Thailand. Southeast Asian J Trop Med Public Health 2012;43:795-807.

24. Haarni I, Hautamaki L. Life experience and alcohol: 60-75 year olds' relationship to alcohol in theme interviews. Nord Stud Alcohol Drugs 2010;27:241-58.

25. Shankar A, McMunn A, Banks J, Steptoe A. Loneliness, social isolation, and behavioral and biological health indicators in older adults. Health Psychol 2011;30:377-85.

26. Holmes WR, Joseph J. Social participation and healthy ageing: a neglected, significant protective factor for chronic non communicable conditions. Globalization and Health serial on the Internet. 2011. Available from: http://www.globalizationadnhealth.com/content/7/1/43.

27. Walker J, Orpin P, Baynes $\mathrm{H}$, et al. Insights and principles for supporting social engagement in rural older people. Ageing Soc 2013;33:938-63.

28. Cornwell EY, Waite LJ. Measuring social isolation among older adults using multiple indicators from the NSHAP study. J Gerontol B Psychol Sci Soc Sci 2009;64B(Suppl 1):i38-i46.

29. Khan N, Wilkinson TJ, Keeling S. Reasons for changing alcohol use among older people in New Zealand. Australas J Ageing 2006;25:97100 .

30. Alexander F, Duff RW. Social interaction and alcohol use in retirement communities. Gerontologist 1988;28:632-6.

31. Byles J, Young A, Furuya H, Parkinson L. A drink to healthy aging: the association between older women's use of alcohol and their health-related quality of life. J Am Geriatr Soc 2006;54:1341-7.

32. Morgan T, Williamson M, Pirotta M, et al. A national census of medicines use: a 24 hour snapshot of Australians aged 50 years and older. Med J Australia 2012;196:5-53.

33. Tanaka E. Toxicological interactions involving psychiatric drugs and alcohol: an update. J Clin Pharm Ther 2003;28:81-95.

34. Merrick EL, Hodgkin D, Garnick DW, et al. Unhealthy drinking patterns and receipt of preventive medical services for older adults. J Gen Intern Med 2013;23:1741-8.

35. Onen SH, Onen F, Mangeon JP, et al. Alcohol abuse and dependence in elderly emergency department patients. Arch Gerontol Geriatr 2005;41:191-200. 